\title{
METACOMMUTATION OF HURWITZ PRIMES
}

\author{
HENRY COHN AND ABHINAV KUMAR \\ (Communicated by Matthew A. Papanikolas)
}

\begin{abstract}
Conway and Smith introduced the operation of metacommutation for pairs of primes in the ring of Hurwitz integers in the quaternions. We study the permutation induced on the primes of norm $p$ by a prime of norm $q$ under metacommutation, where $p$ and $q$ are distinct rational primes. In particular, we show that the sign of this permutation is the quadratic character of $q$ modulo $p$.
\end{abstract}

\section{INTRODUCTION}

In this paper we study the metacommutation mapping, which we will define shortly. Let $\mathbb{H}=\mathbb{R}+\mathbb{R} i+\mathbb{R} j+\mathbb{R} k$ be the algebra of Hamilton quaternions over $\mathbb{R}$, and let $\mathcal{H}$ be the subring of Hurwitz integers, consisting of the quaternions $a+b i+c j+d k$ for which $a, b, c, d$ are all in $\mathbb{Z}$ or all in $\mathbb{Z}+1 / 2$. Recall that for an element $x=a+b i+c j+d k$, its conjugate $x^{\sigma}$ is $a-b i-c j-d k$ (we reserve the notation $\bar{x}$ for reduction modulo a prime). The reduced 1 norm of $x$ is $\mathrm{N}(x)=x x^{\sigma}=a^{2}+b^{2}+c^{2}+d^{2}$, and the reduced trace of $x$ is $\operatorname{tr}(x)=x+x^{\sigma}=2 a$.

It is well known that $\mathcal{H}$ has a Euclidean division algorithm (on the left or right), and thus every one-sided ideal is principal. An element $P \in \mathcal{H}$ is prime if it is irreducible; i.e., it is not a product of two nonunits in $\mathcal{H}$. We say a left prime ideal $\mathfrak{P}=\mathcal{H} P$ lies over the rational prime $p$ if $p \in \mathfrak{P}$, which is equivalent to $\mathrm{N}(P)=p$. If $p$ is odd, there are exactly $p+1$ Hurwitz primes lying over $p$, up to left multiplication by units (in other words, there are exactly $p+1$ prime left ideals lying over $p$ ), while there is only one Hurwitz prime over $p=2$.

Because of the Euclidean algorithm for $\mathcal{H}$, we may factor any nonzero Hurwitz integer $x$ as

$$
x=P_{1} P_{2} \ldots P_{n},
$$

where $P_{i}$ is a Hurwitz prime of norm $p_{i}$. We say that this is a factorization of $x$ modeled on a factorization of $\mathrm{N}(x)$ as $p_{1} \ldots p_{n}$ (where we pay attention to the order of the rational primes $\left.p_{1}, \ldots, p_{n}\right)$. When $\mathrm{N}(x)$ is square-free, such a factorization is unique up to unit migration. That is, if $p_{1}, \ldots, p_{n}$ are distinct, then every

Received by the editors December 31, 2012 and, in revised form, August 30, 2013.

2010 Mathematics Subject Classification. Primary 11R52, 11R27.

The second author was supported in part by National Science Foundation grants DMS-0757765 and DMS-0952486 and by a grant from the Solomon Buchsbaum Research Fund.

${ }^{1}$ Recall that the norm and trace are "reduced" because the norm and trace of the linear transformation of multiplication by $x$ on $\mathbb{H}$ are $\mathrm{N}(x)^{2}$ and $2 \operatorname{tr}(x)$, respectively, for either left or right multiplication. 
factorization of $x$ modeled on $\mathrm{N}(x)=p_{1} \ldots p_{n}$ is of the form

$$
x=\left(P_{1} u_{1}\right)\left(u_{1}^{-1} P_{2} u_{2}\right) \ldots\left(u_{n-1}^{-1} P_{n}\right),
$$

where $u_{1}, \ldots, u_{n-1}$ are units of $\mathcal{H}$.

However, if we want to look at all possible prime factorizations of $x$, then we must allow for changes in the order of the primes $p_{1}, \ldots, p_{n}$. If $P$ and $Q$ are primes lying over distinct rational primes $p$ and $q$, then $P Q$ has a unique (up to unit migration) factorization $Q^{\prime} P^{\prime}$ modeled on $q p$. This process of switching two adjacent primes in the model was named metacommutation by Conway and Smith in their account of unique factorization for Hurwitz integers (see Chapter 5 of [CS]). They prove that the prime factorization of an arbitrary nonzero Hurwitz integer is unique up to metacommutation, unit migration, and recombination: the process of replacing $P P^{\sigma}$ with $\tilde{P} \tilde{P}^{\sigma}$, where $P$ and $\tilde{P}$ are primes of the same norm. Another exposition of this theorem can be found in $[\mathrm{CP}$.

Conway and Smith comment that the metacommutation problem of determining $Q^{\prime}$ and $P^{\prime}$ given $P$ and $Q$ does not seem to be addressed in the literature [CS p. 61]. Their proof that $Q^{\prime}$ and $P^{\prime}$ exist yields an efficient method for computing them via the Euclidean algorithm, but it provides little insight into the properties of the metacommutation map.

In this paper, we analyze the metacommutation map. Let $p$ be a rational prime and $Q$ a Hurwitz prime of norm $q \neq p$. For every prime $P$ of norm $p$, we can find $Q^{\prime}$ and $P^{\prime}$ of norms $q$ and $p$ satisfying $P Q=Q^{\prime} P^{\prime}$, and the pair $\left(Q^{\prime}, P^{\prime}\right)$ is unique up to unit migration. Thus, $P^{\prime}$ is defined only up to left multiplication by a unit, and the equation

$$
(u P) Q=\left(u Q^{\prime}\right) P^{\prime}
$$

implies that replacing $P$ by a left associate has no effect on $P^{\prime}$. Therefore, metacommutation by $Q$ yields a permutation of the $p+1$ primes lying above $p$. We determine the number of fixed points (i.e., primes $P$ that commute with $Q$ modulo units) and sign of this permutation:

Theorem 1.1. Let $p$ and $q$ be distinct rational primes, let $Q$ be a Hurwitz prime of norm q, and consider the Hurwitz primes of norm $p$ modulo left multiplication by units. Metacommutation by $Q$ permutes these primes, and the sign of the permutation is the quadratic character $\left(\frac{q}{p}\right)$ of $q$ modulo $p$.

If $p=2$, or if $Q$ is congruent to a rational integer modulo $p$, then metacommutation by $Q$ is the identity permutation, and otherwise it has $1+\left(\frac{\operatorname{tr}(Q)^{2}-q}{p}\right)$ fixed points.

The simplest reason why $p$ and $q$ could determine the sign of the permutation would be if they determined its cycle structure. However, the fixed point count shows that this is not true. For example, the metacommutation permutations of the primes of norm 3 by $1+2 i$ and $i+2 j$ have 0 and 2 fixed points, respectively.

Note that when $P$ is a fixed point under metacommutation by $Q$, it is both a left and a right divisor of $P Q=Q^{\prime} P$. See $\mathrm{AA}+$ for an analysis of which Hurwitz integers have common left/right divisors and how many they have.

Theorem 1.1 is trivial to check when $p=2$, so we shall assume from now on that $p$ is odd. 


\section{A Conic associated to the primes above $p$}

Let $P$ be a Hurwitz prime of odd norm $p$, and let $\Pi$ be the reduction $\overline{\mathcal{H} P}$ of the left ideal $\mathcal{H} P$ modulo $p$; it is a left ideal of the quaternion algebra $\overline{\mathcal{H}}=\mathcal{H} / p \mathcal{H}$ over $\mathbb{F}_{p}$. This quaternion algebra is split, since its norm form, being a nondegenerate quadratic form in four variables, has a nontrivial zero. (Recall that it is a standard exercise to show, using the pigeonhole principle, that for $\alpha, \beta \in \mathbb{F}_{p} \backslash\{0\}$, the equation $\alpha x^{2}+\beta y^{2}=1$ has a solution $(x, y) \in \mathbb{F}_{p}^{2}$.)

Therefore $\overline{\mathcal{H}} \cong M_{2}\left(\mathbb{F}_{p}\right)$, and since every finite-dimensional module over the matrix algebra $M_{2}\left(\mathbb{F}_{p}\right)$ is a direct sum of standard modules $\mathbb{F}_{p}^{2}$, we see that $\Pi$ must be even-dimensional. It is not the zero ideal since it contains $\bar{P}$, and it is not the entire algebra since it is annihilated by right multiplication by $\overline{P^{\sigma}}$ (because $P P^{\sigma}=p$ ). Thus, $\Pi$ must be two-dimensional inside the four-dimensional algebra $\overline{\mathcal{H}}$.

In fact, prime ideals in $\mathcal{H}$ lying over $p$ are in one-to-one correspondence with two-dimensional left ideals of $\overline{\mathcal{H}}$. Specifically, taking the inverse image under the reduction map gives a bijection between ideals in $\overline{\mathcal{H}}$ and ideals in $\mathcal{H}$ that contain $p$. Ideals in $\mathcal{H}$ containing $p$ are generated by divisors of $p$, which are $1, p$, and the primes of norm $p$, and only the latter correspond to two-dimensional ideals in $\overline{\mathcal{H}}$. Thus, studying the primes of norm $p$ is equivalent to studying the proper ideals in $\overline{\mathcal{H}}$.

Lemma 2.1. There is a unique nonzero element $t=x i+y j+z k$ of trace zero in $\Pi$, up to scaling.

Proof. The reduced trace gives a linear functional on the two-dimensional space $\Pi$. Therefore its kernel $K=\operatorname{ker}(\operatorname{tr})$ is at least one-dimensional. Furthermore, $K$ cannot be all of $\Pi$, since if $x i+y j+z k \in K \backslash\{0\}$, then at least one of $x, y, z$ is not zero, say $x$. Then multiplying on the left by $i$, we get $-x+y k-z j$, which is in $\Pi$ (because $\Pi$ is a left ideal in $\overline{\mathcal{H}}$ ), but not in $K$. Therefore $K$ has dimension 1 .

Next, we observe that for every $t \in \Pi$, the reduced norm $\mathrm{N}(t) \in \mathbb{F}_{p}$ must be zero, since $t=\overline{h P}$ for some $h \in \mathcal{H}$ and $\mathrm{N}(h P)=\mathrm{N}(h) \mathrm{N}(P)=p \mathrm{~N}(h)$, which vanishes modulo $p$.

Let $C_{p} \subset \mathbb{P}^{2}\left(\mathbb{F}_{p}\right)$ be the conic defined by $x^{2}+y^{2}+z^{2}=0$. Given a prime $P$ of norm $p$, let $t_{P}$ be the element of $\Pi$ from Lemma 2.1 (scaled arbitrarily), and let $c_{P}$ be the corresponding point on the conic $C_{p}$.

Proposition 2.2. The map $P \mapsto c_{P}$ is a bijection between primes lying over $p$ up to left multiplication by units and points on the conic $C_{p}$.

Proof. Because primes lying over $p$ correspond to two-dimensional ideals of $\overline{\mathcal{H}}$, it suffices to deal with those ideals. Given a point $c \in C_{p}$ with homogeneous coordinates $(x, y, z)$, let $\Pi_{c}=\overline{\mathcal{H}}(x i+y j+z k)$. It is a nonzero left ideal and is not the full ring $\overline{\mathcal{H}}$, because every element $u \in \Pi_{c}$ satisfies $u(x i+y j+z k)=0$. This describes the inverse map and establishes the bijection.

Since the conic $C_{p}$ is smooth, it has exactly $p+1$ points. We therefore recover the well-known count of the number of primes above $p$.

Corollary 2.3. Let $p$ be an odd rational prime. Then the number of Hurwitz primes above $p$, up to left multiplication by units, is $p+1$. 
The proof given here is fundamentally the same as that in $[\mathrm{P}$. In Vorlesung 9 of $[\underline{\mathrm{H}}$, Hurwitz develops a similar method to count left ideals in $\overline{\mathcal{H}}$, although he does not phrase it in terms of the conic $C_{p}$. From a more abstract perspective, $C_{p}$ is a Severi-Brauer variety (see $\S 6$ of Chapter $\mathrm{X}$ in $[\mathrm{S}]$ ). For a generalization to Eichler orders in quaternion algebras over number fields, we refer the reader to Théorème II.2.3 in [V] or Lemma 7.2 in [KV].

\section{Metacommutation}

Suppose $P$ and $Q$ are Hurwitz primes lying over distinct rational primes $p$ and $q$, respectively. By metacommutation, there are primes $Q^{\prime}$ and $P^{\prime}$ of norms $q$ and $p$ such that

$$
P Q=Q^{\prime} P^{\prime}
$$

and we would like to understand how they depend on $P$ and $Q$.

It is not difficult to compute $P^{\prime}$ and $Q^{\prime}$ efficiently as follows. Using the Euclidean algorithm, choose $P^{\prime} \in \mathcal{H}$ so that $\mathcal{H} p+\mathcal{H} P Q=\mathcal{H} P^{\prime}$. Then $P^{\prime}$ divides $p$, so its norm is $1, p$, or $p^{2}$. It cannot be 1 since every element of $\mathcal{H} p+\mathcal{H} P Q$ has norm divisible by $p$, and it cannot be $p^{2}$ since $P Q \notin \mathcal{H} p$. Thus, $P^{\prime}$ has norm $p$. Now $P Q \in \mathcal{H} p+\mathcal{H} P Q=\mathcal{H} P^{\prime}$, so there exists $Q^{\prime}$ such that $P Q=Q^{\prime} P^{\prime}$, and $Q^{\prime}$ must have norm $q$.

As explained in the introduction, $P^{\prime}$ is defined only up to left multiplication by a unit, and replacing $P$ by a left associate has no effect on $P^{\prime}$. Therefore, metacommutation by any prime $Q$ of norm $q \neq p$ gives a permutation $\tau_{Q}$ of the $p+1$ primes lying above $p$. In the rest of the paper, we analyze this action.

Note that replacing $Q$ by a right associate $Q u$ composes the permutation $\tau_{Q}$ with the permutation $\gamma_{u}$ sending $P$ to $P u$, because

$$
P Q u=Q^{\prime} P^{\prime} u=Q^{\prime}\left(P^{\prime} u\right),
$$

which implies $\tau_{Q u}=\gamma_{u} \circ \tau_{Q}$. Similarly,

$$
\left(P u^{-1}\right)(u Q)=P Q=Q^{\prime} P^{\prime}
$$

implies $\tau_{u Q} \circ \gamma_{u^{-1}}=\tau_{Q}$ or $\tau_{u Q}=\tau_{Q} \circ \gamma_{u^{-1}}^{-1}=\tau_{Q} \circ \gamma_{u}$.

To understand metacommutation more clearly, it is important to rephrase it in terms of the constructions from the previous section. We will continue to use the notation from that section $\left(\Pi, t_{P}\right.$, etc., and the corresponding versions $\Pi^{\prime}, t_{P^{\prime}}$, etc. for $\left.P^{\prime}\right)$.

Theorem 3.1. For $P^{\prime}$ and $Q^{\prime}$ as above, $t_{P^{\prime}}$ and $(\bar{Q})^{-1} t_{P} \bar{Q}$ are equal modulo scaling by $\mathbb{F}_{p}^{\times}$.

Proof. Because $P Q=Q^{\prime} P^{\prime}$, we see that $\overline{P Q}$ is contained in $\Pi^{\prime}$, which is a left ideal. Therefore any left multiple of $\overline{P Q}$ is also in $\Pi^{\prime}$, and hence $\Pi \bar{Q} \subseteq \Pi^{\prime}$. In fact, equality holds, since $\bar{Q}$ is invertible in $\overline{\mathcal{H}}$ and thus both $\Pi \bar{Q}$ and $\Pi^{\prime}$ are twodimensional vector spaces over $\mathbb{F}_{p}$. Finally, since $\bar{Q}$ is invertible and these are left ideals,

$$
\bar{Q}^{-1} \Pi \bar{Q}=\Pi \bar{Q}=\Pi^{\prime} .
$$

In particular, since $t_{P} \in \Pi$, we must have $(\bar{Q})^{-1} t_{P} \bar{Q} \in \Pi^{\prime}$. However, the vector $(\bar{Q})^{-1} t_{P} \bar{Q}$ has trace zero and is nonzero, and therefore it must equal $t_{P^{\prime}}$ up to scaling. 
Now suppose that $t_{P}=x i+y j+z k$ and $\bar{Q}=a+b i+c j+d k$ with $\bar{q}=$ $a^{2}+b^{2}+c^{2}+d^{2}$. Then explicit calculation shows that $(\bar{Q})^{-1} t_{P} \bar{Q}=x^{\prime} i+y^{\prime} j+z^{\prime} k$ with

$$
\left(\begin{array}{l}
x^{\prime} \\
y^{\prime} \\
z^{\prime}
\end{array}\right)=\frac{1}{\bar{q}}\left(\begin{array}{ccc}
a^{2}+b^{2}-c^{2}-d^{2} & 2 a d+2 b c & -2 a c+2 b d \\
-2 a d+2 b c & a^{2}+c^{2}-b^{2}-d^{2} & 2 a b+2 c d \\
2 a c+2 b d & -2 a b+2 c d & a^{2}+d^{2}-b^{2}-c^{2}
\end{array}\right)\left(\begin{array}{l}
x \\
y \\
z
\end{array}\right) .
$$

(Note that the above matrix is the standard parametrization of $S O(3)$ by $S^{3}$ via the double cover $S U(2) \rightarrow S O(3)$.) Let us denote this linear transformation by $\phi_{Q}$. Note that $\phi_{Q}$ lies in $S O_{3}\left(\mathbb{F}_{p}\right)$. This is not hard to see conceptually: $\phi_{Q} \in$ $O_{3}\left(\mathbb{F}_{p}\right)$ because conjugation preserves the quadratic form $v v^{\sigma}$ on quaternions $v$, and $\operatorname{det}\left(\phi_{Q}\right)=1$ because left or right multiplication by a quaternion $v$ has determinant $\mathrm{N}(v)^{2}$ on $\overline{\mathcal{H}}$ (so conjugation has determinant 1 as a linear transformation on $\overline{\mathcal{H}}$ and hence also on the purely imaginary subspace, since the identity element of $\overline{\mathcal{H}}$ is fixed).

The vector $(b, c, d)$ is an eigenvector of $\phi_{Q}$ with eigenvalue 1 (unless it is the zero vector, in which case $\phi_{Q}$ is the identity transformation), since $b i+c j+d k$ obviously commutes with $\bar{Q}$. We are especially interested in eigenvectors that lie on the cone $K_{p}$ given by $x^{2}+y^{2}+z^{2}=0$, as these correspond to fixed points of the permutation $\tau_{Q}$. Note that the eigenvector $(b, c, d)$ lies on the cone $K_{p}$ iff $\bar{q}=a^{2}$.

Lemma 3.2. The characteristic polynomial of $\phi_{Q}$ is

$$
(x-1)\left(x^{2}+2\left(1-\frac{2 a^{2}}{\bar{q}}\right) x+1\right) .
$$

It follows that the eigenvalue 1 has multiplicity greater than 1 iff $\bar{q}=a^{2}$, and the eigenvalue -1 occurs iff $a=0$, in which case it occurs with multiplicity 2 . The simplest way to prove Lemma 3.2 is by direct computation using the matrix for $\phi_{Q}$, but that is cumbersome. As an alternative, we give a coordinate-free proof.

Proof. We begin with the observations that 1 is always an eigenvalue and that $\operatorname{det}\left(\phi_{Q}\right)=1$. To determine the characteristic polynomial, all we need to show is that

$$
\operatorname{tr}\left(\phi_{Q}\right)=\frac{4 a^{2}}{\bar{q}}-1
$$

Equivalently, on the four-dimensional space $\overline{\mathcal{H}}$, the map $v \mapsto(\bar{Q})^{-1} v \bar{Q}$ should have trace $4 a^{2} / \bar{q}$, because the identity element of $\overline{\mathcal{H}}$ is an eigenvector with eigenvalue 1 . Let $u=b i+c j+d k$ be the imaginary part of $\bar{Q}$, so $\bar{Q}=a+u$ and $(\bar{Q})^{-1}=(a-u) / \bar{q}$. Then we must show that the trace of $v \mapsto(a+u) v(a-u)$ on $\overline{\mathcal{H}}$ is $4 a^{2}$. We can write

$$
(a+u) v(a-u)=a^{2} v+a(u v-v u)-u v u .
$$

The map $v \mapsto a^{2} v$ has trace $4 a^{2}$, and the maps $v \mapsto u v$ and $v \mapsto v u$ have trace $2 \operatorname{tr}(u)=0$. Thus, all that remains is to show that $v \mapsto u v u$ has trace 0. Call this map $\varphi$. We will construct an invertible map $\psi$ that anticommutes with $\varphi$, so that $\psi \varphi \psi^{-1}=-\varphi$ and hence $\operatorname{tr}(\varphi)=0$. Specifically, we set $\psi(v)=u^{\prime} v$, where $u^{\prime}$ is a nonzero imaginary quaternion that anticommutes with $u$. To see that such a $u^{\prime}$ exists, note that $v \mapsto u v+v u$ sends imaginary quaternions $v$ to scalars, since it is easy to check that $(u v+v u)^{\sigma}=u v+v u$. Thus, it has a nontrivial kernel, so $u^{\prime}$ exists and $\operatorname{tr} \varphi=0$, as desired. 


\section{FIXXED POINTS}

We now discuss the fixed points of the permutation $\phi_{Q}$, considered as a projective transformation. In particular, we will be most interested in those fixed points that lie on the conic $C_{p}$ defined by $x^{2}+y^{2}+z^{2}=0$. In other words, we are interested in eigenvectors of $\phi_{Q}$ that lie on the cone $K_{p} \subset \mathbb{F}_{p}^{3}$ given by the same equation.

Lemma 4.1. Let $v$ be an eigenvector of $\phi_{Q}$ of eigenvalue $\lambda$. If $\lambda \neq \pm 1$, then $v$ lies on the cone $K_{p}$.

Proof. In terms of the inner product defined by the quadratic form $\langle v, v\rangle=v v^{\sigma}$, we have

$$
\lambda^{2}\langle v, v\rangle=\langle\lambda v, \lambda v\rangle=\left\langle\phi_{Q}(v), \phi_{Q}(v)\right\rangle=\langle v, v\rangle,
$$

with the last equality because $\phi_{Q}$ is an orthogonal transformation.

Thus, the study of the fixed points falls naturally into the following cases.

Case 1A: $b=c=d=0$. In this case, $\phi_{Q}$ is the identity transformation.

Case 1B: $\bar{q}=a^{2}$ but $(b, c, d) \neq(0,0,0)$. The characteristic polynomial of $\phi_{Q}$ is $(x-1)^{3}$; i.e., the eigenvalue 1 occurs with multiplicity 3 . Also, the vector $(b, c, d)$ is on the cone $K_{p}$, since

$$
a^{2}=\bar{q}=a^{2}+b^{2}+c^{2}+d^{2} .
$$

Note that $v_{0}=b i+c j+d k$ commutes with $\bar{Q}$. We claim that $\phi_{Q}$ has no other eigenvectors up to scaling. Because the eigenvalue must be 1 , an eigenvector corresponds to a purely imaginary quaternion that commutes with $\bar{Q}$ and hence also with $v_{0}$. If $x$ were another eigenvector linearly independent of $v_{0}$, then $1, x$, and $v_{0}$ would generate an abelian subalgebra of $\overline{\mathcal{H}}$ of dimension at least three, which is impossible.

Case 2: $a=0$. Then $b^{2}+c^{2}+d^{2}=\bar{q} \neq 0$, so the eigenvector $(b, c, d)$ of eigenvalue 1 is not on the cone $K_{p}$. The eigenvalue -1 occurs with multiplicity 2 , so it is not immediately clear that $\phi_{Q}$ is semisimple. We can see that the $(-1)$-eigenspace is two-dimensional, as follows: the equation $\bar{Q} x=-x \bar{Q}$ is equivalent to $\operatorname{tr}(x \bar{Q})=0$, since $x$ and $\bar{Q}$ are purely imaginary. The linear functional $x \mapsto \operatorname{tr}(\bar{Q} x)$ has at least a two-dimensional kernel in the three-dimensional space of imaginary quaternions, which proves our assertion.

It still remains to be seen whether these eigenvectors are in the cone $K_{p}$. The points in the cone $K_{p}$ and the (-1)-eigenspace are given by the simultaneous solutions of the two equations

$$
\begin{aligned}
& b x+c y+d z=0, \\
& x^{2}+y^{2}+z^{2}=0 .
\end{aligned}
$$

In the projective plane, this is the intersection of a line and a conic, so there are two solutions over the algebraic closure $\overline{\mathbb{F}}_{p}$ if we count with multiplicity. Over the field $\mathbb{F}_{p}$ itself, there are either zero or two solutions: the number of solutions is not one, because the line is not tangent to the conic (it would be tangent iff $b^{2}+c^{2}+d^{2}=0$ ).

In fact, a simple discriminant calculation shows that the solutions are defined over $\mathbb{F}_{p}$ if and only if $-\left(b^{2}+c^{2}+d^{2}\right)$ is a square in $\mathbb{F}_{p}$. Thus, there are two distinct points of intersection when $\left(\frac{-q}{p}\right)=1$ and none when $\left(\frac{-q}{p}\right)=-1$, because $b^{2}+c^{2}+d^{2}=\bar{q}$. 
Case 3: $a \neq 0$ and $\bar{q} \neq a^{2}$. The latter inequality implies $b^{2}+c^{2}+d^{2} \neq 0$, and so $(b, c, d)$ is not on the cone $K_{p}$. The quadratic part of the characteristic polynomial,

$$
x^{2}+2\left(1-\frac{2 a^{2}}{\bar{q}}\right) x+1,
$$

has two distinct roots in $\overline{\mathbb{F}}_{p}$, not equal to \pm 1 . The discriminant of this polynomial is $-16 a^{2}\left(\bar{q}-a^{2}\right) / \bar{q}^{2}$, and so there are two eigenvalues in $\mathbb{F}_{p}$ if $\left(\frac{a^{2}-q}{p}\right)=1$ and none if $\left(\frac{a^{2}-q}{p}\right)=-1$. Note that the corresponding eigenvectors are distinct from each other and from $(b, c, d)$, since they belong to distinct eigenvalues not equal to \pm 1 . Thus, there are either two or zero fixed points on the cone according to the quadratic character of $a^{2}-\bar{q}$, by Lemma 4.1.

We sum up these observations in the following theorem, which unifies the different cases and is the second part of Theorem 1.1

Theorem 4.2. Let $Q$ be a prime of norm $q$, with $\bar{Q}=a+b i+c j+d k \in \overline{\mathcal{H}}$. If $b=c=d=0$, then $\tau_{Q}$ is the identity permutation. Otherwise, $\tau_{Q}$ has $1+\left(\frac{a^{2}-q}{p}\right)$ fixed points.

\section{SPECIAL ORThOgONAL GROUPS AND CONICS OVER FINITE FIELDS}

Let $p$ be an odd prime and $F$ a nondegenerate binary quadratic form over $\mathbb{F}_{p}$. In this section, we gather some general results about $S O(F)$ and its action on affine plane conics given by $F(x, y)=u$. We begin by recalling a few well-known results.

Lemma 5.1. Every nondegenerate binary quadratic form $F(x, y)$ is equivalent over $\mathbb{F}_{p}$ to $x^{2}-t y^{2}$ for some $t \in \mathbb{F}_{p}$.

Proof. First, note that since $p$ is odd, we may diagonalize the form, so we may assume $F(x, y)=\alpha x^{2}+\beta y^{2}$ for some $\alpha, \beta \in \mathbb{F}_{p}$. Since $\alpha$ and $\beta$ are both nonzero, the form represents 1 by the pigeonhole principle. Since it has discriminant $\alpha \beta$, it must be equivalent to the diagonal form $x^{2}+\alpha \beta y^{2}$.

From now on, we work with the diagonal form $g_{t}(x, y)=x^{2}-t y^{2}$, where $t \in$ $\mathbb{F}_{p} \backslash\{0\}$.

Lemma 5.2. Let $u \in \mathbb{F}_{p} \backslash\{0\}$. The number of points on the affine conic $x^{2}-t y^{2}=u$ is $p-\left(\frac{t}{p}\right)$.

Proof. The projective conic has $p+1$ points, since it is isomorphic to $\mathbb{P}^{1}\left(\mathbb{F}_{p}\right)$, and the number of points at infinity is either 2 or 0 , according to whether $t$ is or is not a square modulo $p$.

Proposition 5.3. The group $S O\left(g_{t}\right)$ is cyclic of order $p-\left(\frac{t}{p}\right)$. Every element of $S O\left(g_{t}\right)$ is semisimple.

Proof. The group $O\left(g_{t}\right)$ consists of $2 \times 2$ matrices whose columns are orthonormal with respect to $g_{t}$. If the first column is $(\alpha, \beta)$, with $\alpha^{2}-t \beta^{2}=1$, then the second must be proportional to $(\beta t, \alpha)$ and the constant of proportionality must be \pm 1 . For the matrix to be in $S O\left(g_{t}\right)$, that constant must be 1 . Thus, the elements of $S O\left(g_{t}\right)$ are the matrices of the form

$$
M_{\alpha, \beta}=\left(\begin{array}{cc}
\alpha & \beta t \\
\beta & \alpha
\end{array}\right)
$$


with $\alpha^{2}-t \beta^{2}=1$. By Lemma [5.2, the size of the group is as claimed.

Multiplication of matrices in $S O\left(g_{t}\right)$ leads to the group law

$$
M_{\alpha, \beta} M_{\gamma, \delta}=M_{\alpha \gamma+\beta \delta t, \alpha \delta+\beta \gamma} .
$$

If $t=r^{2}$ is a square, then $\alpha^{2}-\beta^{2} t=\alpha^{2}-\beta^{2} r^{2}=(\alpha-\beta r)(\alpha+\beta r)$. It is easy to see that the map $\eta: S O\left(g_{t}\right) \rightarrow \mathbb{F}_{p}^{\times}$given by $\eta\left(M_{\alpha, \beta}\right)=\alpha+\beta r$ is an isomorphism of groups. Since $\mathbb{F}_{p}^{\times}$is cyclic of order $p-1$, so is $S O\left(g_{t}\right)$.

If $t$ is not a square, consider the map $\eta: S O\left(g_{t}\right) \rightarrow \mathbb{F}_{p^{2}}^{\times}$given by $\eta\left(M_{\alpha, \beta}\right)=$ $\alpha+\beta \sqrt{t}$. It gives an isomorphism of $S O\left(g_{t}\right)$ with the subgroup $N$ of elements of $\mathbb{F}_{p^{2}}^{\times}$of norm 1 . These are the solutions of $z^{p+1}=1$ in $\mathbb{F}_{p^{2}}^{\times}$, so they are $p+1$ in number. Since $N$ is a finite subgroup of the multiplicative group of a field, it is also cyclic.

Note that in either case, we mapped $M_{\alpha, \beta}$ to one of its eigenvalues (either $\alpha+\beta r$ or $\alpha+\beta \sqrt{t}$ ). In fact, in the first case $r$ is a square root of $t$, so both maps are essentially the same.

To show that every element of $S O\left(g_{t}\right)$ is semisimple, observe that the characteristic polynomial of $M_{\alpha, \beta}$ is

$$
x^{2}-2 \alpha x+1,
$$

which has discriminant $4\left(\alpha^{2}-1\right)$. If $\alpha \neq \pm 1$, then the eigenvalues in $\mathbb{F}_{p^{2}}$ are distinct, so $M_{\alpha, \beta}$ is semisimple. If $\alpha= \pm 1$, then $\alpha^{2}-t \beta^{2}=1$ forces $\beta=0$, and therefore $M_{\alpha, \beta}$ is either the identity or its negation. Alternatively, one may use the fact that $S O\left(g_{t}\right)$ is a group of order $p \pm 1$, which is coprime to the characteristic, to deduce semisimplicity of its elements.

Proposition 5.4. Let $\psi \in S O\left(g_{t}\right)$ have characteristic polynomial $x^{2}+(2-v) x+1=$ $(x+1)^{2}-v x$, with $v \neq 0$. Then $v$ is a square in $\mathbb{F}_{p}$ iff

$$
\frac{\left|S O\left(g_{t}\right)\right|}{\operatorname{ord}(\psi)}
$$

is even.

Proof. First, note that since $\psi$ is semisimple, its order is equal to the order of either of its two eigenvalues, which are reciprocals of each other. Therefore $\left|S O\left(g_{t}\right)\right| / \operatorname{ord}(\psi)$ is even iff an eigenvalue $\lambda$ is the square of an element in the corresponding cyclic group $\mathbb{F}_{p}^{\times}$or $N$, with the notation from the proof of the previous proposition.

First, suppose that $t=r^{2}$ is a square, so $S O\left(g_{t}\right) \cong \mathbb{F}_{p}^{\times}$. Because the characteristic polynomial is $(x+1)^{2}-v x$, we have

$$
v=\frac{(\lambda+1)^{2}}{\lambda}
$$

which implies $v$ is a square in $\mathbb{F}_{p}^{\times}$iff $\lambda$ is as well.

Next, suppose $t$ is not a square, so $S O\left(g_{t}\right) \cong N$. If $\lambda=\mu^{2}$ with $\mu \in N$, then

$$
v=\lambda+\frac{1}{\lambda}+2=\mu^{2}+\frac{1}{\mu^{2}}+2=\left(\mu+\frac{1}{\mu}\right)^{2}
$$

and $\mu+\mu^{-1}=\mu+\mu^{p}=\operatorname{tr}(\mu) \in \mathbb{F}_{p}$. Therefore $v$ is a square in $\mathbb{F}_{p}^{\times}$. Conversely, if $v=w^{2}$ is a square, then let $\mu$ be a square root of $\lambda$ in $\overline{\mathbb{F}}_{p}$. We need to show that 
$\mu \in N$. The above calculation shows that

$$
\mu+\frac{1}{\mu}= \pm w
$$

and therefore $\mu$ satisfies a quadratic equation

$$
\mu^{2} \mp w \mu+1=0 .
$$

Thus $\mu \in \mathbb{F}_{p^{2}}$ and $\mu$ has norm 1 . It follows that $\mu \in N$, so $\lambda$ is a square in $N$.

Lemma 5.5. Let $u \neq 0$, and let $D_{t, u}$ be the affine conic given by $x^{2}-t y^{2}=u$. Then $S O\left(g_{t}\right)$ acts simply transitively on $D_{t, u}$.

Proof. The characteristic polynomial of $M_{\alpha, \beta}$ in the variable $x$ is $x^{2}-2 \alpha x+1$, so 1 is an eigenvalue if and only if $\alpha=1$, in which case $\alpha^{2}-t \beta^{2}=1$ implies $\beta=0$ and hence $M_{\alpha, \beta}$ is the identity matrix. Thus, no element of $S O\left(g_{t}\right)$ except the identity matrix can fix any nonzero vector. Because $S O\left(g_{t}\right)$ preserves $D_{t, u}$ and $\left|S O\left(g_{t}\right)\right|=\left|D_{t, u}\right|$, it must act simply transitively.

Corollary 5.6. Let $\psi \in S O\left(g_{t}\right)$ have characteristic polynomial $x^{2}+(2-v) x+1=$ $(x+1)^{2}-v x$, with $v \neq 0$. Then the sign of the permutation induced by $\psi$ on $D_{t, u}$ is $\left(\frac{v}{p}\right)$.

Proof. Let $k=\operatorname{ord}(\psi)$ and $\ell=\left|S O\left(g_{t}\right)\right| / k$. Since $S O\left(g_{t}\right)$ is cyclic and its action on $D_{t, u}$ is simply transitive, the permutation induced on $D_{t, u}$ by any generator of the group is a $\left|S O\left(g_{t}\right)\right|$-cycle. Therefore, the permutation induced by $\psi$ on $D_{t, u}$ is a product of $\ell$ disjoint $k$-cycles, where $k \ell=p \pm 1$ is even. Thus, the sign of the permutation is $(-1)^{(k+1) \ell}=(-1)^{k \ell+\ell}=(-1)^{\ell}$. By Proposition 5.4, this is equal to the quadratic character of $v$ modulo $p$.

\section{Cycle structure And sign of Permutation}

In this section, we analyze the cycle structure of the permutation $\tau_{Q}$ and show that its sign is $\left(\frac{q}{p}\right)$. Once again the analysis splits up into various cases.

Case 1A: $b=c=d=0$. Here $\tau_{Q}$ is the identity permutation, so it has $p+1$ fixed points. The sign of the permutation is 1 , which equals $\left(\frac{q}{p}\right)$ since $q$ is a square $\bmod p$, because $\bar{q}=a^{2}$.

Case 1B: $\bar{q}=a^{2}$ but $(b, c, d) \neq(0,0,0)$. In this case, $\tau_{Q}$ has exactly one fixed point on the conic $C_{p}$, so there remain $p$ points which it must permute. Note that $N=\phi_{Q}-I$ has eigenvalue zero with multiplicity three, so it is nilpotent; in fact, by the Cayley-Hamilton theorem, $N^{3}=0$, and since $p \geq 3$, we have $N^{p}=0$. Then $\phi_{Q}^{p}=(I+N)^{p}=I+N^{p}=I$, since we are in characteristic $p$. Therefore the orbit sizes must all divide $p$. This forces the remaining $p$ points to be in one orbit, since $p$ is prime. The permutation is thus a $p$-cycle, with $\operatorname{sign}(-1)^{p+1}=1$, which is again the same as $\left(\frac{q}{p}\right)$.

For the remaining cases, we make the following observations. Let $v_{0}=(b, c, d)$ be an eigenvector with eigenvalue 1 and nonzero norm. If $v=(x, y, z)$ is a vector on the cone $K_{p}$ and orthogonal to $v_{0}$, then

$$
\begin{aligned}
& b x+c y+d z=0, \\
& x^{2}+y^{2}+z^{2}=0 .
\end{aligned}
$$


As we saw in the analysis of Case 2 in Section 4 , this system has zero or two solutions up to scaling. In the latter case, both are eigenvectors for $\phi_{Q}$ with eigenvalue -1 . These fixed points contribute nothing to the sign of the permutation, so it suffices to look at the orbits of vectors that are not orthogonal to $v_{0}$.

Now if $v$ is a vector on the cone $K_{p}$ not orthogonal to $v_{0}$ (there are $p \pm 1$ of these up to scaling), then we may fix a scaling for $v$ by replacing it by $v /\left\langle v, v_{0}\right\rangle$. Thus we may assume that all these $v$ satisfy $\left\langle v, v_{0}\right\rangle=1$. The linear transformation $\phi_{Q}$ permutes these vectors, for if $\left\langle v_{0}, v\right\rangle=1$, then

$$
\left\langle\phi_{Q}(v), v_{0}\right\rangle=\left\langle\phi_{Q}(v), \phi_{Q}\left(v_{0}\right)\right\rangle=\left\langle v, v_{0}\right\rangle=1 \text {. }
$$

For any such $v$, its projection along $v_{0}$ is the vector $w_{0}=v_{0} /\left\langle v_{0}, v_{0}\right\rangle$, and its projection orthogonal to $v_{0}$ is $v_{\perp}=v-w_{0}$. Note that

$$
\left\langle v_{\perp}, v_{\perp}\right\rangle=\langle v, v\rangle-\left\langle w_{0}, w_{0}\right\rangle=0-\left\langle w_{0}, w_{0}\right\rangle=-\frac{1}{\left\langle v_{0}, v_{0}\right\rangle} .
$$

Conversely, given any vector $w$ of norm $-1 /\left\langle v_{0}, v_{0}\right\rangle$ orthogonal to $v$, there is a unique vector $v$ on the cone $K_{p}$ with $\left\langle v, v_{0}\right\rangle=1$ and $v^{\perp}=w$, namely $v=w+w_{0}$.

Because $v_{0}$ is fixed, $\phi_{Q}$ induces an orthogonal transformation on the orthogonal complement of $v_{0}$. The quadratic form on this subspace has discriminant $1 /\left\langle v_{0}, v_{0}\right\rangle$ and must therefore be equivalent to the diagonal form $f_{Q}(x, y)=x^{2}-t y^{2}$, where $t=-\left\langle v_{0}, v_{0}\right\rangle^{-1}=-\left(b^{2}+c^{2}+d^{2}\right)^{-1}=\left(a^{2}-\bar{q}\right)^{-1}$.

Therefore, $\phi_{Q}$ gives rise to an element $\psi_{Q} \in S O_{2}\left(f_{Q}\right)$, whose characteristic polynomial is

$$
x^{2}+2\left(1-\frac{2 a^{2}}{\bar{q}}\right) x+1
$$

by Lemma 3.2. To understand the orbits of $\phi_{Q}$ on the projectivization of the cone $K_{p}$, it is enough to understand the action of $\psi_{Q}$ on the vectors of norm $u=-1 /\left\langle v_{0}, v_{0}\right\rangle$ in $\mathbb{F}_{p}^{2}$.

Case 2: $a=0$. In this case, the map $\psi_{Q}$ is simply multiplication by -1 , so the permutation breaks up into $\frac{1}{2}\left(p-\left(\frac{-q}{p}\right)\right)$ disjoint transpositions. Because

$$
\begin{aligned}
\frac{1}{2}\left(p-\left(\frac{-q}{p}\right)\right) & =\frac{1}{2}\left(p-\left(\frac{-1}{p}\right)\right)+\frac{1}{2}\left(\frac{-1}{p}\right)\left(1-\left(\frac{q}{p}\right)\right) \\
& \equiv \frac{1}{2}\left(1-\left(\frac{q}{p}\right)\right) \quad(\bmod 2),
\end{aligned}
$$

we see that the sign of the permutation is equal to $\left(\frac{q}{p}\right)$.

Case 3: $a \neq 0$ and $\bar{q} \neq a^{2}$. We apply Corollary [5.6, with $t=a^{2}-\bar{q}$ and $v=4 a^{2} / \bar{q}$, to see that the sign of the permutation is the quadratic character of $4 a^{2} / \bar{q}$ modulo $p$, i.e., $\left(\frac{q}{p}\right)$.

We have thus proved the following theorem.

Theorem 6.1. Let $p$ and $q$ be distinct primes, with $p$ odd. The sign of the permutation induced on the $p+1$ Hurwitz primes of norm $p$ (up to left multiplication by units) through metacommutation by a prime of norm $q$ is $\left(\frac{q}{p}\right)$.

This completes the proof of Theorem 1.1. Note that the cycle structure of the permutation is somewhat more subtle: it is determined by $a, \bar{q}$, and the order of the roots of $x^{2}+2\left(1-2 a^{2} / \bar{q}\right) x+1$ in the multiplicative group $\mathbb{F}_{p^{2}}^{\times}$. 


\section{ACKNOWLEDGEMENTS}

The authors thank Noam Elkies, Benedict Gross, Bjorn Poonen, and the anonymous referee for helpful comments.

\section{REFERENCES}

[AA+] Mohammed Abouzaid, Jarod Alper, Steve DiMauro, Justin Grosslight, and Derek Smith, Common left- and right-hand divisors of a quaternion integer, J. Pure Appl. Algebra 217 (2013), no. 5, 779-785, DOI 10.1016/j.jpaa.2012.08.003. MR3003303

[CP] Boyd Coan and Cherng-tiao Perng, Factorization of Hurwitz quaternions, Int. Math. Forum 7 (2012), no. 41-44, 2143-2156. MR2967414

[CS] John H. Conway and Derek A. Smith, On quaternions and octonions: their geometry, arithmetic, and symmetry, A K Peters Ltd., Natick, MA, 2003. MR.1957212(2004a:17002)

$[\mathrm{H}] \quad$ Adolf Hurwitz, Vorlesungen über die Zahlentheorie der Quaternionen, Verlag von Julius Springer, Berlin, 1919.

[KV] Markus Kirschmer and John Voight, Algorithmic enumeration of ideal classes for quaternion orders, SIAM J. Comput. 39 (2010), no. 5, 1714-1747, DOI 10.1137/080734467. MR:2592031(2011c:11171)

[P] Cherng-tiao Perng, Factorization of Lipschitz quaternions, preprint, 2011.

[S] Jean-Pierre Serre, Local fields, Graduate Texts in Mathematics, vol. 67, Springer-Verlag, New York, 1979. Translated from the French by Marvin Jay Greenberg. MR554237 (82e:12016)

[V] Marie-France Vignéras, Arithmétique des algèbres de quaternions (French), Lecture Notes in Mathematics, vol. 800, Springer, Berlin, 1980. MR/580949 (82i:12016)

Microsoft Research New England, One Memorial Drive, Cambridge, Massachusetts 02142

E-mail address: cohn@microsoft.com

Department of Mathematics, Massachusetts Institute of Technology, Cambridge, MassachusetTs 02139

E-mail address: abhinav@math.mit.edu 